\title{
The effects of gel-based manicure on pulse oximetry
}

\author{
Jia Lin Jacklyn $\underline{\text { Yek }}^{1}$, MBBS, MRCs, Hairil Rizal $\underline{\text { Abdullah }}^{1}$, MBBS, MMed, June Pheck Suan $\underline{G o h}^{1}$, MBBS, FRCA, \\ Yew Weng $\underline{\mathrm{Chan}^{1}}$, MMed, FAMS
}

INTRODUCTION Pulse oximetry is the standard monitoring technique of functional oxygen saturation $\left(\mathrm{SpO}_{2}\right)$. As the use of fingernail polish has been described to alter $\mathrm{SpO}_{2}$ readings, its removal is commonly recommended prior to measurement. Gel-based manicures have gained popularity in recent years due to their attractiveness and longevity. However, the removal of gel nail polish requires a specialised procedure. Valuable time and resources can be saved if removal can be avoided. To our knowledge, there are no available studies on the effect of gel-based manicures on pulse oximetry readings. Hence, we evaluated the effect with two oximeters, using different technology and wavelength combinations.

METHODS 17 healthy female adult volunteers were recruited for this single-blind randomised controlled trial. Subjects with hypothermia, hypotension, poor plethysmographic waveform and nail pathology were excluded. Colours tested were: black, purple, navy blue, green, light blue, white, yellow, orange, pink and red. Pulse oximetry was measured at 15- and 30-second intervals using two different pulse oximeters, the Philips M1191BL and Masimo SET ${ }^{\circledR}$. Means were compared using paired $t$-tests.

RESULTS Using the Masimo oximeter, light blue $(\Delta \mathrm{M}=0.97 \% \pm 0.96 \% ; \mathrm{p}=0.001)$ and orange $(\Delta \mathrm{M}=0.76 \pm 1.17 \%$; $\mathrm{p}=0.016$ ) gel nail polish resulted in a statistically significant increase from baseline $\mathrm{SpO}_{2}$ readings. With the Philips oximeter, the limits of agreement ranged from $2 \%$ for pink to $17 \%$ for black, indicating imprecision.

CONCLUSION Gel-based manicures can result in overestimations of actual readings, delaying detection of hypoxaemia. Gel nail polish should be routinely removed or an alternative monitoring technique sought.

Keywords: gel manicure, nail polish, oxygen saturation, pulse oximetry, $\mathrm{SpO}_{2}$

\section{INTRODUCTION}

Pulse oximetry is a simple, continuous, non-invasive and reliable monitoring device in routine medical practice that measures functional oxygen saturation $\left(\mathrm{SpO}_{2}\right)$ in arterial blood. This inexpensive method also provides heart rate measurements and an indication of tissue perfusion based on pulse amplitude. However, multiple factors can affect the $\mathrm{SpO}_{2}$ reading. Endogenous factors such as low perfusion (secondary to hypothermia, low cardiac output, increased systemic vascular resistance, profound anaemia, etc) and dyshaemoglobinaemia (e.g. carboxyhaemoglobinaemia and methaemoglobinaemia) can compromise $\mathrm{SpO}_{2}$ readings..$^{(1,2)}$ Exogenous factors such as excessive ambient light or motion, intravenous dyes and nail polish may also cause artefactual readings. ${ }^{(2)}$

As the use of fingernail polish has been described to alter the $\mathrm{SpO}_{2}$ reading in normal subjects, removal of the polish is commonly recommended prior to measurement. Gel-based manicures have gained popularity in recent years due to their attractiveness and resistance to chipping, scratching and denting (Fig. 1). ${ }^{(3)}$ Gel nail polish is a type of acrylic-based nail polish composed of a mixture of acrylate monomers that require ultraviolet light for polymerisation and hardening. ${ }^{(3)}$ However, removing gel nail polish is difficult and requires a specialised procedure. In addition, patients are often resistant to removal, especially of elaborate, costly designs. As clinicians increasingly encounter such patients in emergency and operative situations, the question arises of whether gel-based manicures interfere with measurements.
To our knowledge, no studies on gel-based manicure have been conducted. In addition, the literature has shown conflicting results regarding regular ${ }^{(4-7)}$ and acrylic ${ }^{(1)}$ nail polish. Studies by Coté et al ${ }^{(4)}$ described the effects of black, blue and green fingernail polish to significantly lower $\mathrm{SpO}_{2}$ readings by $3 \%-6 \%$, postulating that it could be due to the differing spectrophotometric absorption abilities of the colours. Similarly, Rubin ${ }^{(5)}$ discovered that a particular blue fingernail polish with similar spectrophotometric data resulted in a decrease in $\mathrm{SpO}_{2}$, from $97 \%$ to $87 \%$. Contrastingly, studies by Hinkelbein et $\mathrm{a}^{\mathrm{l}^{(6)}}$ and Yamamoto et $\mathrm{a}^{\left({ }^{(7)}\right.}$ did not find any difference in hypoxic subjects. Valuable time, resources and money can be saved if removal can be avoided. Hence, we evaluated the effects of the gel-based manicure on pulse oximetry readings with two common oximeters, using different technology and wavelength combinations.

\section{METHODS}

This was a randomised single-blind self-controlled study of healthy subjects, conducted at Singapore General Hospital, Singapore. The study was reviewed and approved by an independent ethics committee (CIRB ref no. 2016/2382) and adheres to the applicable EQUATOR CONSORT guidelines. ${ }^{(8)}$

We recruited 17 healthy female adult volunteers in October 2016 after obtaining written informed consent. Male participants were considered for this study but were not included due to the lack of male volunteers. All subjects were aged 21-40 years, of American Society of Anesthesiologists Class 1 and of Asian descent. Subjects with a history of anaemia or

${ }^{1}$ Department of Anaesthesiology, Singapore General Hospital, Singapore

Correspondence: Dr Jacklyn Jia Lin Yek, Medical Officer, Department of Anaesthesiology, Singapore General Hospital, Outram Road, Singapore 169608. jacklyn.yek@mohh.com.sg 
haemoglobinopathy, or who were found to have hypothermia $\left(\mathrm{T}<34^{\circ} \mathrm{C}\right)$, systolic blood pressure $<90 \mathrm{mmHg}$ and/or diastolic blood pressure $<50 \mathrm{mmHg}$, poor plethysmographic waveform or any nail pathology were excluded. A normal plethysmographic waveform was defined visually as consisting of equally spaced, equally wide waves of equal amplitude over 10 seconds, while a poor waveform consisted of an inconsistent or irregular pattern of varying amplitude and/or wavelength. A baseline $\mathrm{SpO}_{2}$ reading on each finger was taken at 30 seconds.

Each subject was assigned a number from 1 to 10 by simple randomisation using an online number generator. ${ }^{(9)} 10$ nail polish colours were pre-selected and numbered from 1 to 10 in the following order: (1) black; (2) purple; (3) navy blue; (4) green; (5) light blue; (6) white; (7) yellow; (8) orange; (9) pink; and (10) red. The fingernails of our subjects were painted from the left to the right hand, beginning with the left little finger to the left thumb (i.e. left little finger, ring finger, middle finger and index thumb) and continuing from the right thumb to right little finger (i.e. right thumb, index finger, middle finger, ring finger and little finger). The aforementioned colour sequence would commence from the finger corresponding to the randomly allocated number, starting the count from the left little finger (Fig. 1).

Pulse oximetry measurements were made with two pulse oximeters: the Philips M1191BL adult finger sleeve sensor (Philips Medizin Systeme Böblingen $\mathrm{GmbH}$, Böblingen, Germany) and the Masimo SET $®$ Wrap-around-Style Sensor (Masimo Corporation, Irvine, CA, USA), connected to the Masimo Root ${ }^{\circledR}$ monitor. The Philips reusable sensor probe emits light at wavelengths of $660 \mathrm{~nm}$ and $940 \mathrm{~nm}$, while the Masimo RD SET ${ }^{\mathrm{TM}}$ Wrap-around-Style Sensor device emits light at $660 \mathrm{~nm}$ and $905 \mathrm{~nm}$. These two models were selected as they were (a) commonly used in our clinical setting; and (b) would illustrate the effects of different wavelength and technology combinations.

Due to the variety of gels available, it was impractical to test the composition of all variations. In this trial, Gellyfit (Sizuka Korea Co Ltd, Seoul, Korea), a gel nail polish, was used. A nail technician coated each nail with four layers of nail polish: a base coat, two layers of gel nail polish and a top coat. LED (light-emitting diode) light was used to cure the nail polish between applications.

All $\mathrm{SpO}_{2}$ recordings were taken in room air with the subjects sitting in the same place to minimise inaccuracies caused by motion artefacts or ambient light. $\mathrm{SpO}_{2}$ recordings were taken before and after nail polish application so that the patient's unpainted finger could be taken as its own control. This was done because previous studies had elicited variations in perfusion rates between the fingers of each individual, resulting in varying $\mathrm{SpO}_{2}$ readings. Results were recorded by a blinded observer who was unaware of which subject was being studied and what nail polish colour, if any, had been applied.

The pulse oximetry probe was placed directly on the nail bed. Measurements of each finger were recorded after stable readings were obtained, at predetermined intervals of 15 and 30 seconds. The time interval for $\mathrm{SpO}_{2}$ recording was determined based on signal averaging time, which was set at 10 seconds for both devices, and time efficiency in conducting the study. Data dropout was defined

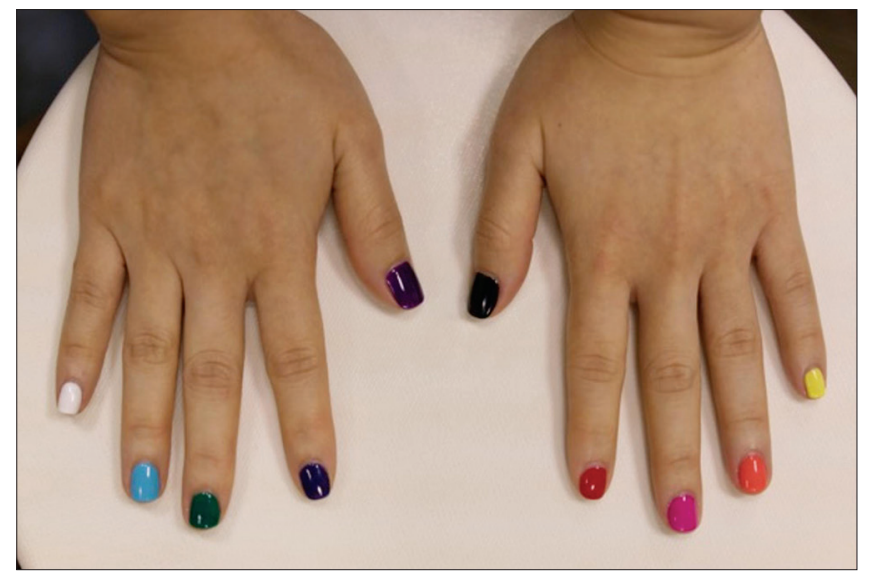

Fig. 1 Photograph of a volunteer's fingernails (assigned number: 5) shows gel-based manicure of ten different colours.

as instances where a poor pulsatile $\mathrm{SpO}_{2}$ waveform was seen after two minutes of repositioning and manual stabilisation of the $\mathrm{SpO}_{2}$ probe, based on a notation made by the independent observer.

For the Philips oximeter, no reading could be obtained in six instances, for one black, two purple and three pink nails, and these were treated as missing values in the data analysis. For the Masimo oximeter, a poor waveform was present in 14 readings at the 15 -second interval, with an accompanying $\mathrm{SpO}_{2}$ reading. In these instances, the values obtained were included in the analysis.

Sample size was derived on a basis of a paired $t$-test. To achieve a power of 0.80 , a Type 1 error rate of $5 \%$, an effect size of 0.70 and a standard deviation of $1 \%$ in the difference between pre- and post-application $\mathrm{SpO}_{2}$ readings, a minimum of 16 volunteers were needed. Results were not stratified according to age, as previous studies have shown that age does not affect $\mathrm{SpO}_{2}$ readings. Dropout values were replaced using a single imputation method of mean substitution, where missing values were replaced with the mean value obtained from all readings of that finger using a single imputation of missing values. ${ }^{(10)}$ Statistical analysis was performed using paired independent $t$-test. A p-value $<0.05$ was considered statistically significant. Standard deviation was used to assess variability in $\mathrm{SpO}_{2}$ readings. Precision of $\mathrm{SpO}_{2}$ readings was analysed using the Bland-Altman method of comparison on the $95 \%$ limits of agreement, which were estimated by calculating the mean difference \pm 1.96 standard deviation of the differences. ${ }^{(11)}$ F-ratio test was used to compare the standard deviation of mean readings, using a statistical significance level of 0.05 . Statistical analysis was performed with IBM SPSS Statistics for Windows version 23.0 (IBM Corp, Armonk, NY, USA).

\section{RESULTS}

The mean systolic blood pressure was 111 (range 95-128) mmHg and mean diastolic blood pressure was 70 (range 54-88) $\mathrm{mmHg}$. The mean tympanic temperature was $36.5^{\circ} \mathrm{C}$ (range $35.8^{\circ} \mathrm{C}-37.1^{\circ} \mathrm{C}$ ). The effects of light blue $(\Delta \mathrm{M}=0.97 \% \pm 0.96 \%$ [range $-1.93 \%$ to $-0.01 \%] ; p=0.001)$ and orange $(\Delta M=0.76 \% \pm 1.17 \%$ [range $-1.93 \%$ to $0.41 \%$ ]; $p=0.016$ ) gel-based nail polish on Masimo pulse oximeters revealed a statistically significant increase in baseline pulse oximeter readings. White $(\Delta M=0.06 \%$ 
Table I. Functional oxygen saturation readings of gel-based manicure colours compared with controls $(\mathrm{n}=17)$.

\begin{tabular}{|c|c|c|c|c|c|c|c|}
\hline \multirow[t]{3}{*}{ Colour } & \multicolumn{7}{|c|}{ Masimo oximeter } \\
\hline & \multicolumn{3}{|c|}{ Mean \pm standard deviation } & \multirow[t]{2}{*}{ F-ratio } & \multicolumn{2}{|c|}{ Limits of agreement } & \multirow[t]{2}{*}{ p-value } \\
\hline & Before nail polish $\left(M_{B}\right)$ & After nail polish $\left(M_{A}\right)$ & Apparent change $\left(M_{A}-M_{B^{\prime}} \%\right)$ & & Lower & Upper & \\
\hline Black & $98.65 \pm 1.00$ & $98.29 \pm 1.98$ & $-0.35 \pm 2.36$ & $3.9^{*}$ & -4.28 & 4.99 & 0.55 \\
\hline Purple & $98.59 \pm 0.35$ & $98.15 \pm 0.70$ & $-0.44 \pm 2.12$ & $4.0^{*}$ & -3.71 & 4.60 & 0.40 \\
\hline Navy blue & $98.35 \pm 0.36$ & $97.68 \pm 0.86$ & $-0.68 \pm 3.84$ & $6.4^{*}$ & -6.85 & 8.20 & 0.48 \\
\hline Green & $98.88 \pm 0.32$ & $99.06 \pm 0.35$ & $0.18 \pm 1.85$ & 1.2 & -3.79 & 3.44 & 0.70 \\
\hline Light blue & $98.76 \pm 0.28$ & $99.73 \pm 0.13$ & $0.97 \pm 0.96$ & $4.6^{*}$ & -2.85 & 0.91 & $0.00^{+}$ \\
\hline White & $98.76 \pm 0.25$ & $98.82 \pm 0.66$ & $0.06 \pm 2.57$ & $7.0^{*}$ & -5.09 & 4.97 & 0.93 \\
\hline Yellow & $98.65 \pm 0.24$ & $99.00 \pm 0.26$ & $0.35 \pm 1.25$ & 1.2 & -2.80 & 2.09 & 0.26 \\
\hline Orange & $98.35 \pm 0.31$ & $99.12 \pm 0.31$ & $0.76 \pm 1.17$ & 1.0 & -3.07 & 1.54 & $0.02^{+}$ \\
\hline Pink & $98.59 \pm 0.35$ & $98.79 \pm 0.25$ & $0.21 \pm 1.84$ & 2.0 & -3.81 & 3.40 & 0.65 \\
\hline Red & $98.59 \pm 0.17$ & $99.06 \pm 0.20$ & $0.47 \pm 1.21$ & 1.4 & -2.86 & 1.92 & 0.13 \\
\hline \multirow[t]{3}{*}{ Colour } & \multicolumn{7}{|c|}{ Philips oximeter } \\
\hline & \multicolumn{3}{|c|}{ Mean \pm standard deviation } & F-ratio & \multicolumn{2}{|c|}{ Limits of agreement } & p-value \\
\hline & Before nail polish $\left(P_{B}\right)$ & After nail polish $\left(P_{A}\right)$ & Apparent change $\left(P_{A}-P_{B^{\prime}} \%\right)$ & & Lower & Upper & \\
\hline Black & $99.59 \pm 0.87$ & $98.32 \pm 4.39$ & $-1.26 \pm 4.58$ & $25.5^{*}$ & -7.71 & 10.24 & 0.27 \\
\hline Purple & $99.65 \pm 0.15$ & $99.71 \pm 1.67$ & $0.59 \pm 0.86$ & $123.0^{*}$ & -1.75 & 1.63 & 0.78 \\
\hline Navy blue & $99.59 \pm 0.15$ & $99.76 \pm 0.10$ & $0.18 \pm 0.61$ & 2.3 & -1.37 & 1.02 & 0.25 \\
\hline Green & $99.41 \pm 0.27$ & $99.76 \pm 0.16$ & $0.35 \pm 1.10$ & $2.8^{*}$ & -2.51 & 1.80 & 0.21 \\
\hline Light blue & $99.71 \pm 0.21$ & $99.76 \pm 0.16$ & $0.06 \pm 0.88$ & 1.6 & -1.79 & 1.67 & 0.79 \\
\hline White & $99.76 \pm 0.11$ & $99.85 \pm 0.07$ & $0.09 \pm 0.53$ & $2.5^{*}$ & -1.14 & 0.96 & 0.51 \\
\hline Yellow & $99.71 \pm 0.17$ & $99.91 \pm 0.05$ & $0.21 \pm 0.71$ & $11.6^{*}$ & -1.59 & 1.18 & 0.25 \\
\hline Orange & $99.59 \pm 0.19$ & $99.15 \pm 0.56$ & $-0.44 \pm 2.50$ & $8.7^{*}$ & -4.46 & 5.34 & 0.48 \\
\hline Pink & $99.65 \pm 0.17$ & $99.82 \pm 0.09$ & $0.18 \pm 0.50$ & $3.6^{*}$ & -1.15 & 0.80 & 0.16 \\
\hline Red & $99.76 \pm 0.11$ & $99.71 \pm 0.14$ & $-0.06 \pm 0.79$ & 1.6 & -1.49 & 1.60 & 0.76 \\
\hline
\end{tabular}

${ }^{*}$ Calculated F-ratio is larger than the critical value of $\mathrm{F} . \mathrm{tp}<0.05$ is statistically significant.

$\pm 2.57 \%$ [range $-2.51 \%$ to $2.63 \%$ ]; $p=0.93$ ) had the smallest effect of all the colours used (Table I).

Although the Philips pulse oximeter did not produce statistically significant findings, a few of the results were clinically relevant. Black $(\Delta \mathrm{P}=-1.26 \% \pm 4.58 \%$ [range $-3.32 \%$ to $5.84 \%$ ]; $\mathrm{p}=0.27)$ and orange $(\Delta \mathrm{P}=-0.44 \% \pm 2.50 \%$ [range $-2.06 \%$ to $2.94 \%$ ]; $\mathrm{p}=0.48)$ gel-based manicure had a mean bias of more than \pm $2 \%$. Light blue $(\Delta \mathrm{P}=0.06 \% \pm 0.88 \%$ [range $0.82 \%$ to $0.94 \%$ ] $\mathrm{p}=0.79$ ) had the smallest effect of all the colours used (Table I). With the Philips oximeter, the limits of agreement ranged from $2 \%$ for pink nail polish, indicating a small variation in all readings, to $17 \%$ for black nail polish, indicating a greater variation in $\mathrm{SpO}_{2}$ readings. The limits of agreement ranged from 3\% for light blue nail polish to $9 \%$ for black nail polish using the Masimo oximeter.

When the F-test was used to compare the standard deviation of readings as a measure of precision, the colours black $(F=3.9)$, purple $(F=4.0)$, navy blue $(F=6.4)$, light blue $(F=4.6)$ and white $(F=7.0)$ were above the critical $F$-value, indicating a wider range of readings on the Masimo device after application of nail polish. Comparatively, the colours black $(F=25.5)$, purple $(F=123.0)$, green $(F=2.8)$, white $(F=2.5)$, yellow $(F=11.6)$, orange $(F=8.7)$ and pink $(F=3.6)$ showed comparatively poorer precision on the Philips device after application of nail polish. Notably, a wider range of F-ratio, and therefore a larger variance, was seen on the Philips oximeter $(F=1.6-123.0)$ than the
Masimo oximeter $(\mathrm{F}=1.0-7.0)$. In addition, black $(\mathrm{F}=25.5)$ and purple $(F=123.0)$ affected the precision of the readings on the Philips device much more than the other colours.

\section{DISCUSSION}

To the best of our knowledge, our study is the first to evaluate the accuracy and precision of pulse oximetry on gel-based manicure. We found that black nail polish resulted in poorer precision in $\mathrm{SpO}_{2}$ readings for both the Philips and Masimo models (Figs. 2a \& b). This was consistent with the existing literature. ${ }^{(4-7)}$ However, it was also noted that orange and light blue gel nails resulted in a statistically significant increase in $\mathrm{SpO}_{2}$ readings on the Masimo device, findings that were vastly different from those in the literature. Yamamoto et $\mathrm{al}^{(7)}$ postulated that due to their specific light-absorption characteristics, various polish preparations and colours do not correlate with human eye colour perception; the hypothesis was further confirmed by spectrophotometric studies performed by Rodden et al. ${ }^{(12)}$ As colour perceived by the human eye is due to the summation of various absorption values within the visible range of light, ${ }^{(13)}$ it does not correlate with light absorption at the wavelengths emitted by sensor probes, and hence may account for our findings.

Moreover, our findings suggest that nail polish could result in an overestimation of actual oxygen saturation. The chromatic 

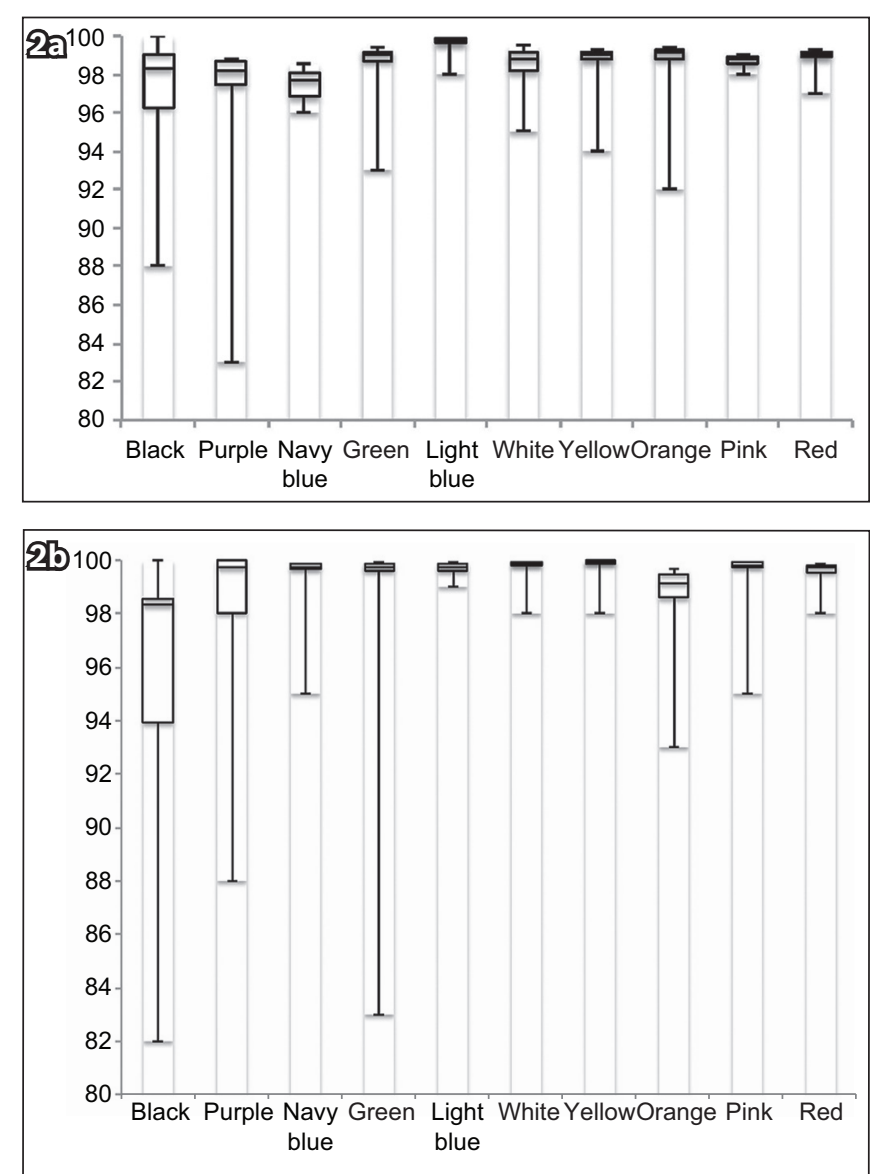

Fig. 2 Box-whisker plots show functional oxygen saturation findings using (a) the Masimo SET ${ }^{\circledR}$ and (b) the Philips M1191BL after application of gel nail polish.

characteristics of nail polish determine its light absorption and scattering interactions, resulting in optical shunting of light around the finger periphery. The effect may be more prominent in the Masimo model with its brighter LED light. ${ }^{(13)}$ Furthermore, the effect of optical shunting could be compounded by the different wavelengths of each sensor probe. Importantly, although our findings were statistically significant, they were not clinically significant $(<2 \%)$.

The Philips oximeter failed to detect readings $1.8 \%$ of the time (six out of 340 readings) as compared to the Masimo oximeter, which detected all readings. In addition, the Philips device was associated with poorer precision in readings. This could be due to the differences between the Philips Fourier artifact-suppression technology (FAST) and the Masimo signal extraction technology (SET). ${ }^{(13)}$ SET uses a discrete saturation transformer with a reference signal generator, an adaptive filter and a peak picker, which work in concert to determine the most likely $\mathrm{SpO}_{2}$ value based on all incoming signals. ${ }^{(12)}$ Conversely, the FAST algorithm identifies the frequency components of the pulse rate and compares those to the frequency components of the incoming signal to select the component that is at pulse rate for both the red and infrared wavelengths, attenuating incoming signals that do not occur synchronously with the pulse rate. ${ }^{(13)}$ Hence, the differences in performance between the two pulse oximeters may be a result of the Masimo SET being superior in separating the arterial signal from venous and other artefactual signals, such as gel nail polish, that may result in a poorer signal-to-noise ratio.
This study was not without limitations. First, our study only involved healthy subjects, who may not be representative of a patient population with significant cardiac or respiratory disease, and lower baseline $\mathrm{SpO}_{2}$. Hypoxic conditions ${ }^{(14)}$ may result in further reductions in accuracy and precision. Without hypoxic subjects, the effects of desaturation on pulse oximetry readings were not investigated. Further studies on hypoxic subjects may be enlightening, as an overestimation of actual oxygen saturation could result in a delay, or even failure, in the detection of hypoxaemia, leading to a disastrous patient outcome. Second, co-oximetry was not used as a measure of accuracy, as it entailed taking arterial blood gas. Spectral analysis of each color was also not quantified with spectrophotometry. Third, single imputation methods were used for $\mathrm{SpO}_{2}$ dropout values, which could be related to the colour of the nails, possibly reducing the variability of our results. Lastly, different brands and thickness of gel nail polish may produce slightly different results. We mitigated this problem by using a commonly used brand of gel nail polish that contains similar compounds with most other brands. The same brand of nail polish was used for all colours in the study. Furthermore, standardising both the nail technician and the number of layers applied minimised any differences in the thickness of the gel nail polish.

In conclusion, different colours of nail polish can cause a statistically significant change in pulse oximeter readings in healthy volunteers, although our findings were not clinically significant. We did not investigate the effects of gel nail polish on hypoxic patients. It can also cause overestimations of actual $\mathrm{SpO}_{2}$ readings, which could delay or even fail in the detection of hypoxaemia. In clinical, surgical and emergency settings, gel nail polish should either be routinely removed or an alternative monitoring technique sought.

\section{REFERENCES}

1. Hinkelbein J, Koehler H, Genzwuerker HV, Fiedler F. Artificial acrylic finger nails may alter pulse oximetry measurement. Resuscitation 2007; 74:75-82.

2. Ralston AC, Webb RK, Runciman WB. Potential errors in pulse oximetry. III: Effects of interference, dyes, dyshaemoglobins and other pigments. Anaesthesia $1991 ; 46: 291-5$.

3. Rieder EA, Tosti A. Cosmetically induced disorders of the nail with update on contemporary nail manicures. J Clin Aesthet Dermatol 2016; 9:39-44.

4. Coté CJ, Goldstein EA, Fuchsman WH, Hoaglin DC. The effect of nail polish on pulse oximetry. Anesth Analg 1988; 67:683-6.

5. Rubin AS. Nail polish color can affect pulse oximeter saturation. Anesthesiology 1988; 68:825.

6. Hinkelbein J, Genzwuerker HV, Sogl R, Fiedler F. Effect of nail polish on oxygen saturation determined by pulse oximetry in critically ill patients. Resuscitation 2007; 72:82-91.

7. Yamamoto LG, Yamamoto JA, Yamamoto JB, Yamamoto BE, Yamamoto PP. Nail polish does not significantly affect pulse oximetry measurements in mildly hypoxic subjects. Respir Care 2008; 53:1470-4.

8. Schulz KF, Altman DG, Moher D; CONSORT Group. CONSORT 2010 statement: updated guidelines for reporting parallel group randomised trials. Int J Surg 2011; 672-7.

9. Urbaniak GC, Plous S. Research Randomizer (version 4.0). Available at: http:// www.randomizer.org/. Accessed June 22, 2013.

10. Zhang Z. Missing data imputation: focusing on single imputation. Ann Transl Med 2016; 4:9.

11. Bland JM, Altman DG. Measuring agreement in method comparison studies. Stat Methods Med Res 1999; 8:135-60.

12. Rodden AM, Spicer L, Diaz VA, Steyer TE. Does fingernail polish affect pulse oximeter readings? Intensive Critical Care Nurs 2007; 23:51-5.

13. Cairo JM, Pilbeam SP. Mosby's Respiratory Care Equipment. 8th ed. St Louis, MO: Mosby/Elsevier, 2010.

14. Severinghaus JW, Spellman MJ Jr. Pulse oximeter failure thresholds in hypotension and vasoconstriction. Anesthesiology 1990; 73:532-7. 